\title{
IMPROVEMENT OF BOTTOM BOUNDARY LAYERS MODELING UNDER INTERACTIONS OF WAVE AND WAVE-INDUCED CURRENT
}

\author{
Jinhai Zheng ${ }^{1}$, Chi Zhang ${ }^{2}$, Yigang Wang ${ }^{3}$ and Zeki Demirbilek ${ }^{4}$
}

\begin{abstract}
The bottom boundary layer characteristics beneath waves transforming on a natural beach are specifically affected both by wave and wave-induced current. This study presents an improved approach for coastal bottom boundary layers modeling under interactions of wave and wave-induced current. The improvement is achieved by formulating the mean horizontal pressure gradient term in the boundary layer equation with wave parameters and mean water level This formulation represents the balance between the wave excess momentum flux gradient and the hydrostatic pressure gradient in a spatially transforming wave field, accounting for the effect of the wave-induced cross-shore current. Model is validated with experimental data for normally incident shoaling wave over a sloping bed. Calculated results agree well with data for instantaneous velocity profiles, wave oscillating amplitudes and mean velocity profiles In particular, model reasonably reproduces the observed local onshore mean flow near the bottom beneath shoaling wave. It is revealed that the proposed formulation of the mean horizontal pressure gradient plays an important role in bottom boundary layer modeling under wave transforming over an variable near-shore bathymetry, and that the present model can be conveniently and reliably coupled with a sediment transport model to study coastal processes in engineering applications.
\end{abstract}

Keywords: wave boundary layer; wave-induce current; wave-current interactions; numerical model

\section{INTRODUCTION}

The coastal bottom boundary layer (BBL) characteristics influence numerous sedimentation problems, the morphological evolution, and the dissipation of surface waves. Coexisting waves and currents in prototype environments affect the structure of the BBL. The source of current may be wave-induced on natural beaches or from rivers or runoff in estuaries. Understanding the mechanics of wave-current coastal BBL has attracted attention of many scientists and engineers for several decades, and the numerical models for this topic have been extensively studied.

The first-order wave-current bottom boundary layer equation is commonly written as:

$$
\frac{\partial u}{\partial t}=\frac{\partial \tilde{u}_{\infty}}{\partial t}-\frac{1}{\rho} \frac{\partial \bar{p}}{\partial x}+\frac{1}{\rho} \frac{\partial \tau}{\partial z}
$$

where $u$ is the instantaneous horizontal velocity, $\tilde{u}_{\infty}$ is the wave-component of the near-bed free stream velocity, $\bar{p}$ is the mean pressure and $\tau$ is the shear stress. In Eq. 1, the current effect appears as a mean horizontal pressure gradient term $-1 / \rho \partial \bar{P} / \partial x$. For different generation mechanisms of current, the treatment of this term may also be different.

Many studies have been conducted to investigate the wave-current BBL above a horizontal bed (e.g., Grant and Madsen 1979; Davies et al. 1988; You et al. 1994; Malarkey and Davies 1998; Guizien et al. 2003; Homeldal et al. 2004; Shi and Wang 2008), where the current was generated independently with and added to the wave, such as generated in oscillatory flow tunnels or wave flumes with recirculation systems (e.g., Bakker and Van Doorn 1978; Kemp and Simons 1982;). Under such conditions, the mean horizontal pressure gradient term has been conveniently neglected assuming that the current-related pressure gradient is very small compared with the wave-related pressure gradient (Malarkey and Davies 1998; Homeldal et al. 2004; Henderson et al. 2004), or formulated as a function of a pre-known reference current velocity independent of wave parameters (Fredsøe and Deigaard 1992; You 1994; Shi and Wang 2008), such as the depth-averaged velocity. For example, Shi and Wang (2008) related the mean pressure gradient term to a shear current velocity calculated empirically by the depth-averaged mean velocity. Their model results show good agreements with the experimental data of Bakker and Van Doorn (1978), in which the current was generated by a

${ }^{1}$ State Key Laboratory of Hydrology-Water Resources and Hydraulic Engineering, Hohai University, 1 Xikang Road, Nanjing, 210098, China

${ }^{2}$ State Key Laboratory of Hydrology-Water Resources and Hydraulic Engineering, Hohai University, 1 Xikang Road, Nanjing, 210098, China

${ }^{3}$ Key Laboratory of Coastal Disaster and Defence of Ministry of Education, Hohai University, 1 Xikang Road, Nanjing, 210098, China

${ }^{4}$ U.S. Army Engineer Research and Development Center, Coastal and Hydraulics Laboratory, 3909 Halls Ferry Road, Vicksburg MS, 39180, USA 
recirculation system over a horizontal bed. Those cases can fairly represent the wave-runoff interactions or the wave-tide interactions, where the current is not generated by the wave and devoid of any strong vertical variation. However, those approaches cannot be directly applied for the wavecurrent interactions over an arbitrary bottom configuration on natural beaches. In this case, it is the wave-induced current that interacts with wave in the BBL, which warrants somewhat different mathematical treatment of the mean horizontal pressure gradient term.

This study focuses on the interactions of wave and wave-induced cross-shore current, such as the undertow. Following reasons can be summarized to explain why a different approach is necessary for this case:

1. The current is driven by wave transformation, as a result the formulation of the mean pressure gradient should have relationships with wave parameters.

2. The current velocity is unknown as a prior, so the external model input of current is no more straightforward.

3. The wave-induced cross-shore current generally exhibits a strong vertical variation both in velocity magnitude and direction through the water column, so it appears difficult to find a reference velocity.

4. The depth-averaged mean cross-shore current velocity should be zero for maintaining mass conservation in a closed coast. In other words, there is a current at any certain elevation in the water column, but there is no current from the perspective of depth-averaging. Therefore, the depth-averaged current velocity cannot be used to estimate the mean horizontal pressure gradient, which is clearly not zero over the depth.

In present study, the formulation of the current-related horizontal pressure gradient term is revisited to consider the effect of the wave-induced cross-shore current on the BBL, with an objective of developing an improved BBL model of general applicability to a wider range of coastal sedimentation problems.

\section{MEHODOLOGY}

\section{Governing Equation}

The wave-averaged form of the Eq. 1 can be written as:

$$
-\frac{1}{\rho} \frac{\partial \bar{p}}{\partial x}=-\frac{1}{\rho} \frac{\partial \bar{\tau}}{\partial z}
$$

Since the boundary layer approximation applies, the mean horizontal pressure gradient is constant though the boundary layer and is equal to the value just outside the boundary layer, and following relationship is obtained:

$$
-\frac{1}{\rho} \frac{\partial \bar{p}}{\partial x}=-\frac{1}{\rho} \frac{\partial \bar{p}_{\infty}}{\partial x}=-\frac{1}{\rho} \frac{\partial \bar{\tau}_{\infty}}{\partial z}
$$

where the subscript $\infty$ means the physical quantity at the outer edge of the boundary layer. It can be seen that the current-related horizontal pressure gradient equals to the vertical gradient of the mean shear stress outside the boundary layer.

Following Svendsen et al. (1987) and Stive and De Vriend (1994), the local wave-averaged horizontal momentum equation at the outer edge of boundary layer under transforming waves can be expressed as:

$$
\frac{\partial \bar{\tau}_{\infty}}{\partial z}=\frac{\partial}{\partial x} \rho \overline{\left(\tilde{u}_{\infty}^{2}-\tilde{w}_{\infty}^{2}\right)}+\frac{\partial}{\partial x} \rho g \bar{\eta}
$$

where $w$ is the vertical component of instantaneous velocity, and $\bar{\eta}$ is the mean surface elevation. The first term in the right side of Eq. 4 represents the cross-shore gradient of wave excess momentum flux due to spatial variation in the wave field, such as wave shoaling or breaking. The second term represents the gradient of hydrostatic pressure resulted from associated wave set-down or set-up. The local imbalance between those two terms will thus result in vertical variations in the mean shear stress and the mean current as well. This concept has been used to solve the wave-averaged cross-shore mean current (e.g., Reniers et al. 2004; Zheng and Tang 2009), but not yet implemented in the wave-current bottom boundary layer models. 
Zheng (2007) derived a set of expressions for the vertical distribution of the wave excess momentum flux through the water column. Under normally incident linear waves and shallow water approximation, the wave excess momentum flux below the wave trough can be given by:

$$
\rho \overline{\left(\tilde{u}_{\infty}^{2}-\tilde{w}_{\infty}^{2}\right)}=\frac{E}{h}=\frac{\rho g H^{2}}{8 h}
$$

where $E, H$ and $h$ are respectively the wave energy density, wave height, and water depth. Combining Eq. 3, 4 and 5, the current-related mean horizontal pressure gradient can be expressed as:

$$
-\frac{1}{\rho} \frac{\partial \bar{p}}{\partial x}=-\frac{g H}{4 h} \frac{\partial H}{\partial x}-g \frac{\partial \bar{\eta}}{\partial x}
$$

As shown, the formulation of the mean horizontal pressure gradient term under transforming wave is a function of wave parameters. The magnitude of this term depends on the relative balance between two competing physical processes: wave excess momentum flux gradient due to wave transformation, and hydrostatic pressure gradient due to the variation of the mean surface level. It could be positive or negative depending on the specific wave-dynamic region, but not necessarily related to any reference current velocity. With this formulation, non-zero values of the current-related horizontal pressure gradient can be obtained even if the depth-averaged current velocity would be zero. Therefore, realistic physical features in the wave and wave-induced current coexisting condition can be reasonably represented. We use the linear wave theory in this study for its simplicity, but any nonlinear wave theory could also be used. The cross-shore distribution of wave height and mean surface elevation can be obtained from measurements or from numerical wave models (Zheng et al. 2008).

Substituting Eq. 6 into Eq. 1 and introducing the Boussinesq approximation $\tau=\rho\left(v_{t}+v\right) \partial u / \partial z$, the improved governing equation for the bottom boundary layer under interactions of wave and waveinduced cross-shore current can be written as:

$$
\frac{\partial u}{\partial t}=\frac{\partial \tilde{u}_{\infty}}{\partial t}-\left(\frac{g H}{4 h} \frac{\partial H}{\partial x}+g \frac{\partial \bar{\eta}}{\partial x}\right)+\frac{\partial}{\partial z}\left[\left(v_{t}+v\right) \frac{\partial u}{\partial z}\right]
$$

Equation 7 considers the spatial variation of wave field and mean surface elevation and accounts for the effects of wave-induced cross-shore currents on the bottom boundary layer. The depth-averaged current velocity, which is commonly necessary input for previous models to calculate the mean pressure gradient, is not required as an input in this model.

\section{Turbulence closure}

A modified low Reynolds number $k-\varepsilon$ model is used to provide the turbulence closure, referred to as the MKM model by Sana et al. (2007). The transport equations for the turbulent kinetic energy $k$ and the turbulent dissipation rate $\varepsilon$ are given by

$$
\begin{gathered}
\frac{\partial k}{\partial t}=\frac{\partial}{\partial z}\left[\left(\frac{v_{t}}{\sigma_{k}}+v\right) \frac{\partial k}{\partial z}\right]+v_{t}\left(\frac{\partial u}{\partial z}\right)^{2}-\varepsilon \\
\frac{\partial \varepsilon}{\partial t}=\frac{\partial}{\partial z}\left[\left(\frac{v_{t}}{\sigma_{\varepsilon}}+v\right) \frac{\partial \varepsilon}{\partial z}\right]+f_{1} C_{\varepsilon 1} \frac{\varepsilon}{k} v_{t}\left(\frac{\partial u}{\partial z}\right)^{2}-f_{2} C_{\varepsilon 2} \frac{\varepsilon^{2}}{k}
\end{gathered}
$$

The turbulent eddy viscosity is calculated by

$$
v_{t}=f_{u} C_{u} \frac{k^{2}}{\varepsilon}
$$

The standard values of model parameters are used, and they are $C_{u}=0.09, C_{\varepsilon 1}=1.4, C_{\varepsilon 2}=1.8, \sigma_{k}=1.4$, $\sigma_{\varepsilon}=1.3, f_{1}=1.0$. Two functions $f_{u}$ and $f_{2}$ are defined according to Sana et al. (2007).

\section{Boundary conditions}

The no-slip condition is applied at the bottom as:

$$
u\left(z_{0}, t\right)=0 \quad\left(z_{0}=k_{s} / 30\right)
$$


where $k_{s}$ is the Nikuradse roughness. The velocity is equal to the free stream at the upper edge of the bottom boundary layer designated as $\mathrm{z}=z_{\max }$, and we have:

$$
u\left(z_{\max }, t\right)=u_{\infty}
$$

The bottom boundary conditions for the turbulent quantities are defined as:

$$
k\left(z_{0}, t\right)=0, \quad \varepsilon\left(z_{0}, t\right)=2 v\left(\frac{\partial \sqrt{k}}{\partial z}\right)^{2}
$$

A zero-flux turbulence condition is employed at the outer boundary of the bottom boundary layer:

$$
\frac{\partial}{\partial z} k\left(z_{\max }, t\right)=0, \quad \frac{\partial}{\partial z} \varepsilon\left(z_{\max }, t\right)=0
$$

\section{Numerical schemes}

A Crank-Nicholson type implicit finite-difference scheme was employed to discretize the governing equations. An iterative algorithm was applied to solve the nonlinear system of differential equations. Closer to the bed, where the vertical gradients are particularly high, a stretched vertical grid was used by allowing grid spacing to increase exponentially in vertical direction. The computational convergence for parameters $u, k$ and $\varepsilon$ was achieved when the maximum relative differences was less than 0.00001 between two consecutive iterations.

\section{RESULTS AND DISCUSSIONS}

Model was tested with a laboratory measurement of bottom boundary layer beneath normally incident shoaling waves over a sloping bed (Case 1, Lin and Hwung, 2002), conducted in a wave flume of $9.5 \mathrm{~m}$ long, $0.3 \mathrm{~m}$ wide and $0.7 \mathrm{~m}$ deep. Monochromatic waves of height $0.053 \mathrm{~m}$ and period $1.41 \mathrm{~s}$ were generated over a smooth bed of 1:15 slope. Time series of the water surface elevation and corresponding horizontal velocity in the bottom boundary layer were collected at 10 cross-shore sections, numbered P1 to P10 with decreasing distance to the breaking point, see Fig. 1.

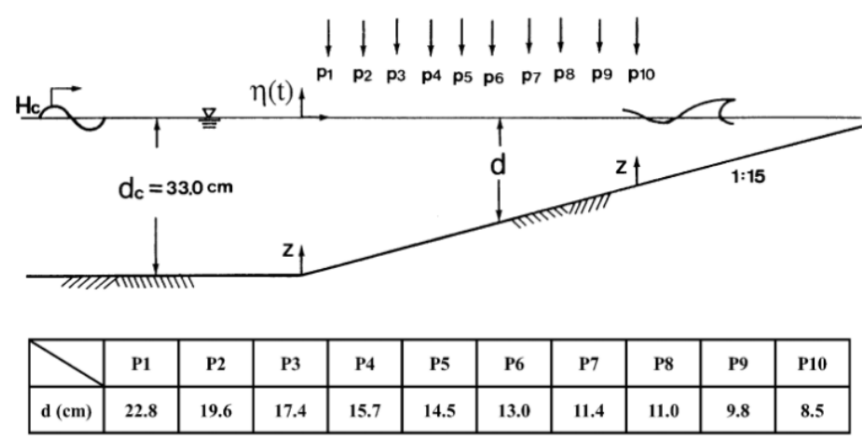

Figure 1. Sketch of wave flume and locations of measurement (Lin and Hwung, 2002).

The measured data from locations P4, P8 and P10 were used to test the present model. The model incident boundary was set to $0.005 \mathrm{~m}$ above the bottom where the time series of free stream velocity $\left(u_{\infty}\right)$ were measured. Calculations were made with 100 vertical grid cells and 300 time steps per wave period, and Nikuradse roughness $\left(k_{s}\right)$ of $0.002 \mathrm{~m}$. The values of calculated mean horizontal pressure gradient at P4, P8 and P10 were $0.002 \mathrm{~m} / \mathrm{s}^{2}, 0.005 \mathrm{~m} / \mathrm{s}^{2}$, and $0.01 \mathrm{~m} / \mathrm{s}^{2}$, respectively. The values of the mean horizontal pressure gradient at those three locations are always positive under shoaling wave and increases with decreasing distance to the breaking point, implying that the onshore hydrostatic pressure gradient induced by set down is greater than the offshore momentum flux gradient caused by increasing wave heights due to shoaling. This trend continues as wave propagating closer to the surf zone, tending to drive an onshore mean current in the bottom boundary layer.

Figure 2 shows the comparisons of instantaneous velocity profiles at eight instants of time (phases) within a wave cycle. The vertical coordinate is normalized by the Stokes Length defined as $\delta=\sqrt{v T / \pi}$. The calculated results with and without the mean horizontal pressure gradient term are drawn by solid lines and dash lines, respectively. Generally good agreements are found. There is a clear offshore-leaning tendency of the upper velocity profile at all test locations. This phenomenon is increasingly apparent while approaching the breaking point which may be due to increasing positive 
current-induced mean pressure gradient, and is well captured by the prediction with the mean pressure gradient. In general, the incorporation of mean pressure gradient produces larger velocities and improves predictions near the bottom during positively accelerating phases (phase A-C).
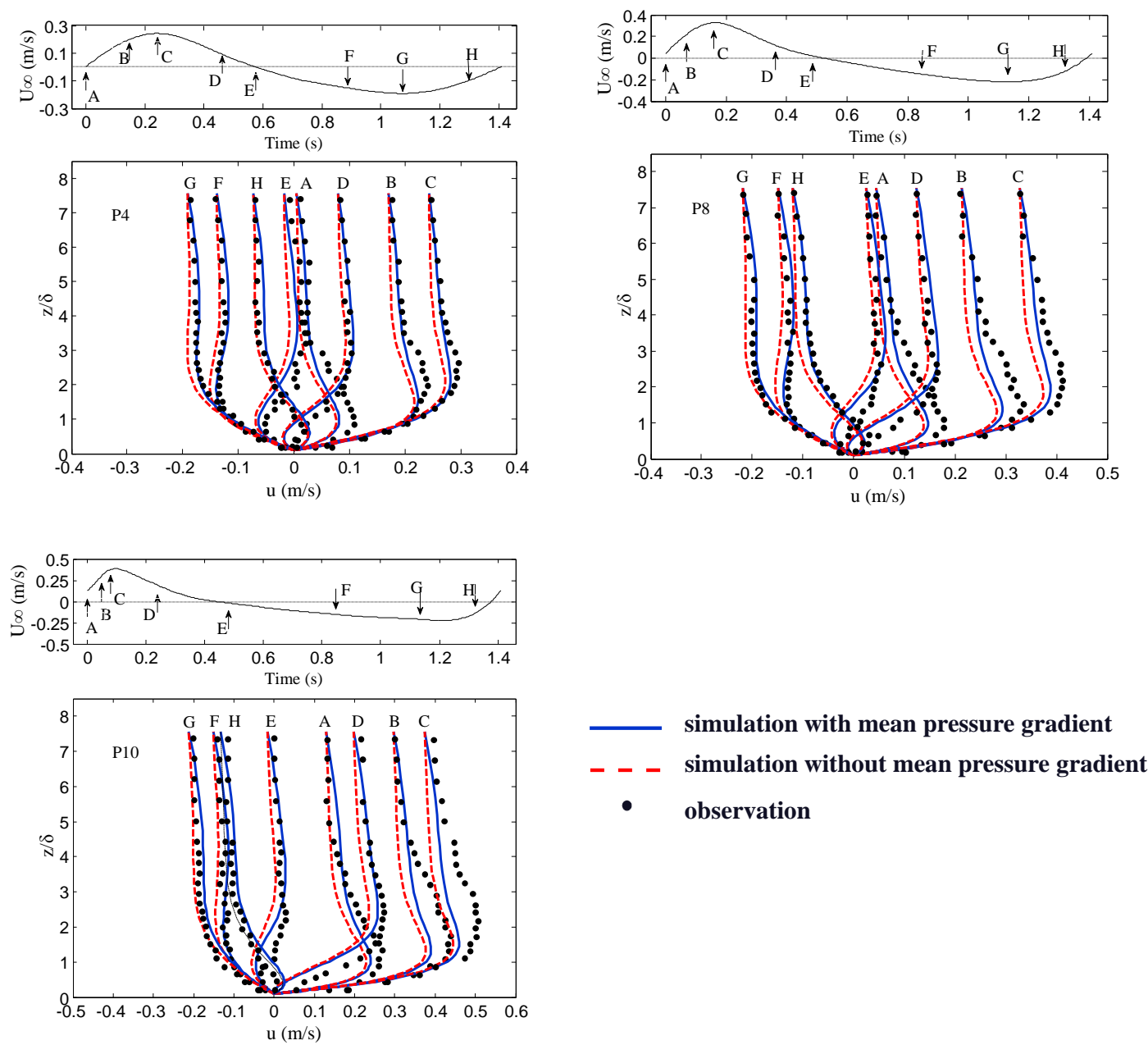

Figure 2. Comparison with the experimental data (Lin and Hwung 2002) at gauges P4, P8 and P10. The top panel shows time series of free stream velocity. The bottom panel shows instantaneous velocity profiles observed (dots) and simulated with (solid lines) and without (dash lines) the mean horizontal pressure gradient.

Figure 3 shows the comparisons of oscillating velocity amplitudes. Agreements are satisfactory and the current-induced mean pressure gradient seems to have little effect on the calculated oscillating velocity amplitudes. The wave boundary layer thickness is under-predicted for positive phases, which may be attributed to the neglect of the advection of breaking wave turbulence from the surf zone.
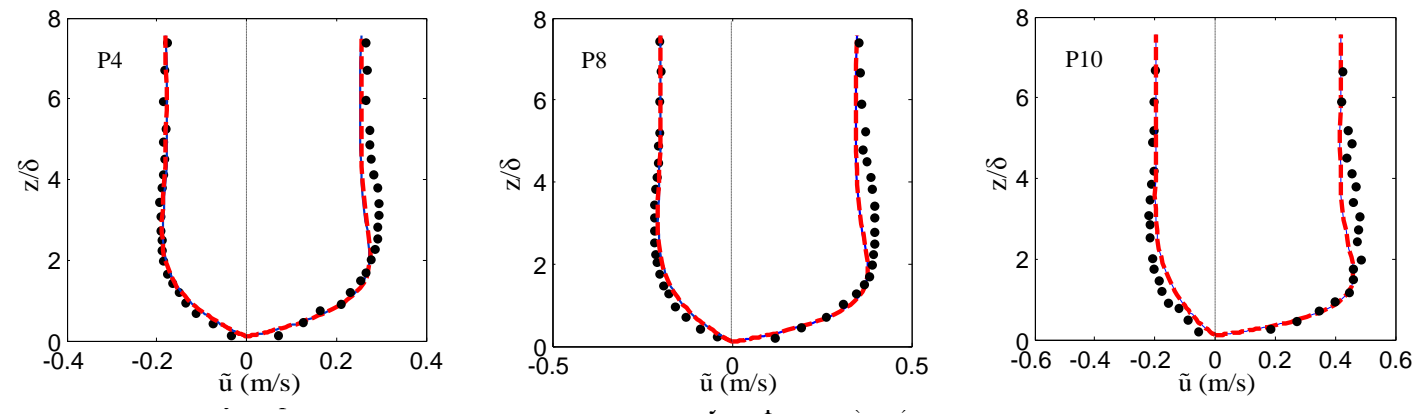

Figure 3. Comparison of oscillating velocity amplitude from observations (dots, from Lin and Hwung 2002) and simulations performed with the mean horizontal pressure gradient (solid lines) and without (dash lines). 
However, the incorporation of the mean pressure gradient term remarkably improves the predictions of the mean velocity profile, shown in Fig. 4. The calculated results with the positive mean pressure gradient reasonably reproduced the observed local onshore mean velocity near the bottom, while the predictions without the mean pressure gradient show to be offshore directed. This improvement is attributed to the improved formulation of the mean horizontal pressure gradient term. The maximum near-bed onshore mean velocity is underestimated, which may be due to the neglect of the boundary layer streaming in present model.

In addition, the mean horizontal pressure gradient further dominates the direction of the mean bottom shear stresses shown in the Table 1. The mean bottom shear stress calculated with the positive mean pressure gradient is positive and increases with decreasing distance to the breaking point, while the calculation results without that pressure gradient show to be offshore directed. This may be meaningful for the simulation of the onshore sediment transport outside the surf zone.
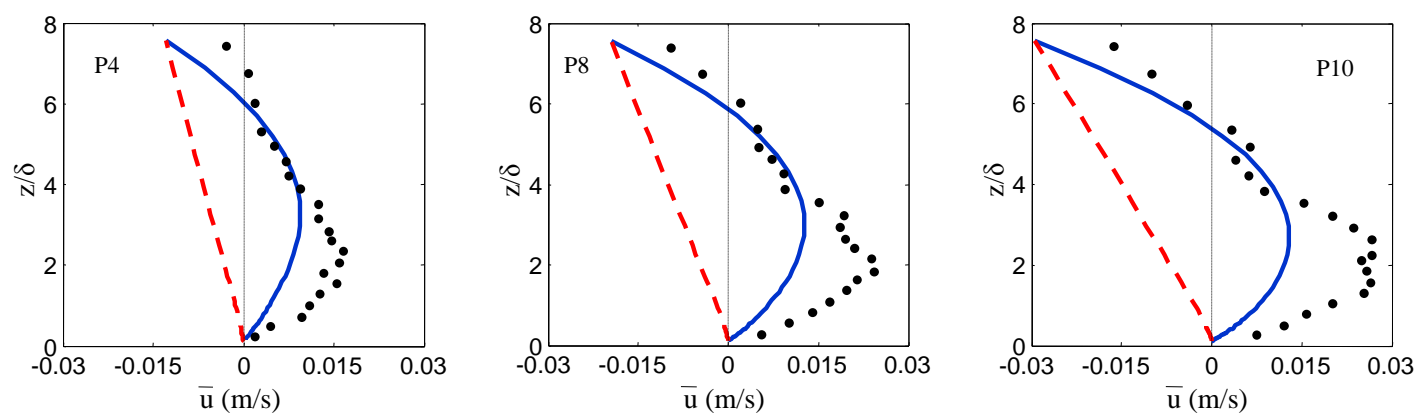

Figure 4. Comparison between data and model calculated mean velocity profiles. The (dots) are data from Lin and Hwung (2002) and simulations are with the mean horizontal pressure gradient (solid lines) and without (dash lines).

\begin{tabular}{|c|c|c|c|}
\hline & $\mathrm{P} 4$ & P8 & $\mathrm{P} 10$ \\
\hline With $-1 / \rho \partial \bar{P} / \partial x$ & 0.0084 & 0.0141 & 0.0178 \\
\hline Without $-1 / \rho \partial \bar{P} / \partial x$ & -0.0026 & -0.004 & -0.0062 \\
\hline
\end{tabular}

\section{CONCLUDING REMARKS}

A formulation for the mean horizontal pressure gradient that deals with the wave-induced crossshore current is developed and implemented in a wave-current bottom boundary layer model. This term is derived from wave-averaged momentum equation, and the magnitude of the mean horizontal pressure gradient depends on the relative balance between the wave excess momentum flux gradient and the hydrostatic pressure gradient. Model-data comparisons show that the proposed formulation fairly improves the bottom boundary layer modeling under interactions of wave and wave-induced current beneath shoaling wave, involving instantaneous velocity profiles, oscillating velocity amplitudes and mean velocity profiles. In particular, model reasonably reproduces the observed local onshore mean current near the bottom. It is revealed that the proposed formulation of the mean horizontal pressure gradient plays an important role in bottom boundary layer modeling under wave transforming over an variable near-shore bathymetry, and that the present model can be conveniently and reliably coupled with a sediment transport model to study coastal processes in engineering applications.

\section{ACKNOWLEDGMENTS}

The work was supported by the National Natural Science Foundation of China (Grant No. 50979033), the Program for New Century Excellent Talents in University of China (NCET-07-0255), and the Special Research Funding of State Key Laboratory of Hydrology-Water Resources and Hydraulic Engineering (Grant No. 2009585812).

\section{REFERENCES}

Bakker, W.T., and T. Van Doorn. 1978. Near-bottom velocities in waves with a current, Proceedings of $16^{\text {th }}$ International Conference on Coastal Engineering, ASCE, 1394-1413. 
Davies, A.G., R.L. Soulsby, and H.L. King. 1988. A numerical model of the combined wave and current bottom boundary layer, Journal of Geophysical Research, 93, 491-508.

Fredsøe, J., and R. Deigaard. 1992. Mechanics of coastal sediment transport, World Scientific, Singapore.

Grant, W.D., and O.S. Madsen. 1979. Combined wave and current interaction with a rough bottom, Journal of Geophysical Research, 84, 1797-1808.

Guizien, K., M. Dohmen-Janssen, and G. Vittori. 2003. 1DV bottom boundary layer modeling under combined wave and current: Tubulent separation and phase lag effects, Journal of Geophysical Research, 108, 3000-3016.

Henderson, S.M., J.S. Allen, and P.A. Newberger. 2004. Nearshore sandbar migration predicted by an eddy-diffusive boundary layer model, Journal of Geophysical Research, 109, doi: 10.1029/2003JC002137.

Holmedal, L.E., D. Myrhaug, and K.J. Eidsvik. 2004. Sediment suspension under sheet flow conditions beneath random waves plus current, Continental Shelf Research, 24, 2065-2091.

Kemp, P.H., and R.R. Simons. 1982. The interaction of waves and a turbulent current: waves propagating with the current, Journal of Fluid Mechanics, 116, 227-250.

Lin, C., and H.H. Hwung. 2002. Observation and measurement of the bottom boundary layer flow in the prebreaking zone of shoaling waves, Ocean Engineering, 29, 1479-1502.

Malarkey, J., and A.G. Davies. 1998. Modeling wave-current interactions in rough turbulent bottom boundary layers, Ocean Engineering, 25, 119-141.

Reniers, A.J.H.M., E.B. Thomton, T.P. Stanton, and J.A. Roelvink. 2004. Vertical flow structure during Sandy Duck: observations and modeling, Coastal Engineering, 51, 237-260.

Sana, A., A.R. Ghumman, and H. Tanaka. 2007. Modification of the damping function in the $k-\varepsilon$ model to analyze oscillatory boundary layers, Ocean Engineering, 34, 320-326.

Shi, J.Z., and Y. Wang. 2008. The vertical structure of combined wave-current flow, Ocean Engineering, 35, 174-181.

Stive, M.J.F., and H.J. De Vriend. 1994. Shear stresses and mean flow in shoaling and breaking waves, Proceedings of $24^{\text {th }}$ International Conference on Coastal Engineering, ASCE, 594-608.

Svendsen, I.A. 1984. Mass flux and undertow in a surf zone, Coastal Engineering, 8, 345-365.

You, Z.J. 1994. A simple model for current velocity profiles in combined wave-current flows, Coastal Engineering, 23, 289-304.

Zheng, J.H. 2007. Depth-dependent expression of obliquely incident wave induced radiation stress, Progress in Natural Science, 17, 1067-1073.

Zheng, J.H., H. Mase, Z. Demirbilek, and L. Lin. 2008. Implementation and evaluation of alternative wave breaking formulas in a coastal spectral wave model, Ocean Engineering, 35, 1090-1101.

Zheng, J.H., and Y. Tang. 2009. Numerical simulation of spatial lag between wave breaking point and location of maximum wave-induced current, China Ocean Engineering, 23, 59-71. 